\title{
CONTROL OF BODY HEIGHT IN A STICK INSECT WALKING ON A TREADWHEEL
}

\author{
H. CRUSE ${ }^{1}$, J. SCHMITZ1 ${ }^{1}$ U. BRAUN ${ }^{2}$ and A. SCHWEINS ${ }^{1}$ \\ ${ }^{1}$ Faculty of Biology, University of Bielefeld, Postfach 100131, D-4800 Bielefeld 1, \\ Germany and ${ }^{2}$ FB Biologie, University of Kaiserslautern, Postfach 3049, D-6750 \\ Kaiserslautern, Germany
}

Accepted 29 April 1993

\begin{abstract}
Summary
The properties of the system that controls the distance between body and ground was investigated in the stick insect Carausius morosus. The insect walked on a lightweight double treadwheel under open-loop or closed-loop conditions. The open-loop investigations show that the dynamic behaviour of the height-control system in the walking animal can be described in terms of a simple proportional system with negligible dynamic properties, and it is therefore much simpler than the height-control system in the standing animal. Under open-loop conditions, we found no coupling between contralateral or ipsilateral legs. This agrees with the findings on standing animals. The force-height characteristic shows two ranges, in each of which the system exhibits a linear relationship but a different slope. Under closed-loop conditions, the force-height characteristic shows the same two linear ranges, but the slopes are greater than under open-loop conditions. Because the height controller of each leg can be considered to act like a spring, this result means that under closed-loop conditions the controller is stiffer than it is under open-loop conditions.
\end{abstract}

\section{Introduction}

When a six-legged animal stands or walks on an uneven surface, the correct distance between body and ground must be maintained by the appropriate cooperation of the six legs. For the standing stick insect, several investigations (Wendler, 1964; Kemmerling and Varju, 1981, 1982) have shown that the animal as a whole acts as a height controller with PD properties, i.e. it is sensitive to position and velocity. Later investigations (Cruse et al. 1989, 1992) showed that each individual leg also acts as an independent height controller and that no neuronal coupling between the control systems of the legs needs to be assumed. Each individual leg acts as a feedback controller whose PD properties have a prominent phasic component; this phasic component has also been found in the resistance reflexes of the individual joints (Bässler, 1983; Schmitz, 1985).

Only indirect evidence exists concerning the mechanisms which control body height in the walking insect. Cruse (1976) measured the body height of a stick insect during

Key words: stick insect, walking, height control, depressor muscle, motor pattern, Carausius morosus. 
walking on irregular surfaces. The data could be explained if each leg was assumed to act as an independent height controller (nonlinear P feedback controller). However, in these free-walking animals, no direct measurement of the height-controlling system was possible.

In this paper, the static and dynamic properties of the height-controlling system of the walking animal are investigated by testing the stick insect on a lightweight double wheel (Graham, 1981). The apparatus was developed to allow for both open-loop and closedloop experiments. In addition to investigating the whole animal, influences between contralateral legs were investigated by using two wheels whose height could be changed independently. The latter experiments were carried out using both force measurements and electrophysiological recordings.

\section{Materials and methods}

Adult female stick insects (Carausius morosus $\mathrm{Br}$.) were used in all experiments. The mass of the animals ranged between 0.8 and $1.0 \mathrm{~g}$. Pattex adhesive was used to fix each animal dorsal side up on a horizontal metal holder by all three thoracic segments. The head and legs were left free to move. A micromanipulator was used to position the holder above a lightweight double treadwheel (one wheel for each side of the body). Each wheel had a diameter of $40 \mathrm{~cm}$ and an inertial moment of $390 \mathrm{~g} \mathrm{~cm}^{-2}$. The equivalent mass of one wheel was approximately $1 \mathrm{~g}$ (Graham, 1981). The common axis of both wheels was fixed to a balance so that the wheels could be easily moved up and down (for details, see Graham, 1981). A force of $0.8 \mathrm{mN}$ was necessary to move the wheels to a height of $10 \mathrm{~mm}$. The vertical inertial load of the whole treadwheel corresponded to about eight times the body mass of the animal because it included the weight of the balance as well as both wheels.

For the open-loop experiments, the wheels were fixed at a given distance from the body (defined as the distance between the ventral margin of the body and the upper margin of the wheels). This distance ranged from -3 to $21 \mathrm{~mm}$. A force transducer (Philips PR 9833) was mounted on one beam of the balance to measure the vertical forces exerted on the treadwheel by the animal. The forces were recorded on a pen recorder (Hellige 218081 ). Because they varied during each walk, they were digitized, and a mean value ( \pm S.D.) was calculated for each walk. The duration of each evaluated walk was about 10s. The first $2 \mathrm{~s}$ after the start of each walk was not evaluated.

In the open-loop experiments, the height of the body was measured in the following way. We determined the distance between the body and wheel at which the mean force was zero. This zero-force distance was different for individual animals. The mean value for all animals was $9 \pm 1.5 \mathrm{~mm}$ ( \pm S.D.), so the zero-force heights of all animals were standardised to $9 \mathrm{~mm}$ for later calculation of common mean values, as shown in the figures.

In the closed-loop experiments, the position of the wheels was not fixed. Instead, the animal could choose the distance between its body and the wheels by moving the wheels up or down. However, the wheels could be loaded with different weights fixed to the beam of the balance. These weights could pull the wheels down (positive force) or up 
(negative force). The height of the wheels was recorded by means of a nearly frictionless method. A copper wire was attached to the axis of the wheels. Its other end was submerged in a small water tank between two metal plates serving as electrodes. The wire and plates were connected into a circuit that formed a potentiometer. Thus, the distance between the wire and the electrodes, and therefore the height of the wheel, was proportional to the voltage measured from the wire (for details, see Cruse and Müller, 1984). The heights, like the forces, varied more or less regularly during the walk. The mean height was calculated from digitized height values of a walking session of approximately $10 \mathrm{~s}$.

To test for contralateral coordination, the two wheels were mounted not on one common axis but on two different ones. The axis of one wheel was fixed, whereas the axis of the other was mounted on a micromanipulator and could be moved up and down by hand. In this case, as in the open-loop experiment, we measured the force exerted by the legs walking on the fixed wheel.

\section{Electrophysiological investigations}

\section{Preparation}

For electrophysiological experiments, the wheels were again mounted on two different axes, but the animal was attached by the ventral surface of its thorax and abdomen (using Pattex adhesive) to a stick of balsawood mounted above the treadwheel so as to allow free movement of all the coxae. The position of each treadwheel could be adjusted to provide good conditions for leg movement during walking. The initial body-to-wheel distance for both sides was adjusted to about $7 \mathrm{~mm}$. The dissection of the animal was similar to the method described by Godden and Graham (1984) and by Schmitz et al. (1991). The mesothoracic and metathoracic terga were opened dorsally by a longitudinal slit, and the tergal halves were slightly bent laterally and fixed with insect pins. The gut was removed to gain access to the mesothoracic ganglion and its lateral nerves. The cavity of the animal was filled with Ringer's solution (Wood, 1957).

\section{Leg movements and extracellular recordings}

Data were obtained from nine animals. The leg movements of a walking animal were monitored in two different ways. (a) The movements of the right middle leg in the horizontal plane were monitored by a photodiode system (von Helversen and Elsner, 1977). This system provided a continuous analogue signal corresponding to the forward and backward movement of the femur, i.e. it monitored movement of the thoraco-coxal joint. (b) The whole animal was filmed from above with a video camera for later evaluation of the walking movements and the coordination of all legs. The videotape was synchronized with the electrophysiological recordings by a single-frame trigger, giving a time resolution of $\pm 20 \mathrm{~ms}$.

During walking, the mesothoracic coxa-trochanter joint is moved downward by the depressor trochanteris muscle. This muscle is subdivided into two parts, which are innervated by different motoneurones lying in separate nerves. One part, the coxal part of the depressor trochanteris muscle, is located entirely inside the coxa (Marquardt, 1940; 
Graham, 1985) and is innervated by nerve C2 (Schmitz, 1986). The second part consists of the thoraco-trochanteral muscles $\mathrm{t}-\mathrm{tr}$ and $\mathrm{p}$-tr (Graham, 1985). They arise from the tergum and pleurum, respectively, span the subcoxal joint and pass down into the coxa, where they attach to the tendon of the coxal part of the depressor trochanteris muscle (Marquardt, 1940). The thoraco-trochanteral muscles are innervated by nerve nl4a (Graham, 1985). To investigate the motor output to the depressor trochanteris muscles, bilateral recordings were made from nl4a using oil-and-hook electrodes (Schmitz et al. 1988). We did not record from nerve C2 because this was not possible without severely obstructing the walking leg. However, the activity of the motoneurones in nerve nl4a appears to represent the motor output to the depressor trochanteris muscle adequately, because electrical stimulation of this nerve elicits considerable force from the depressor muscle (Herrmann and Schmitz, 1989). Investigations of the resistance reflex at the coxa-trochanter joint revealed that the motoneurones in nerve nl4a that have the larger spike amplitudes belong to the thoraco-trochanteral part of the depressor trochanteris muscle (J. Schmitz, unpublished results). Only these large-amplitude spikes were considered in the analysis. The extracellular recordings, together with the leg position signal, the synchronizing video trigger signal and the position signal of the right treadwheel, were stored using a d.c. tape recorder for later evaluation.

\section{Stimulation}

Although the position of the left treadwheel was always kept constant at the initial distance $(7 \mathrm{~mm})$ during an experimental session, the height of the right treadwheel could be varied. The right treadwheel was lowered in $5 \mathrm{~mm}$ steps, held for approximately 10-20s, and then brought back to the initial height. Height changes were completed within $200 \mathrm{~ms}$. Data were obtained only during stimuli in which the animal continued walking for at least $10 \mathrm{~s}$ after the change in height.

\section{Data evaluation}

Selected episodes of recordings of the left and right nerve nl4a, as well as the position signal of the right middle leg and the position signal of the right treadwheel, were digitized (sampling rate $5 \mathrm{kHz}$ ) using a CED1401 interface (Cambridge Electronic Design), stored on disk and evaluated with the aid of a software package (Spike II version 4.02, Cambridge Electronic Design). This software allows discrimination of several spike amplitudes within a recording and provides facilities for various methods of analyzing stimulus-response relationship.

Data were processed in the following way. The treadwheel was held in the initial position and the posterior extreme positions (PEPs) of the right middle leg's steps were determined from the leg position signal. They were marked to serve as trigger points for the construction of phase histograms. The duration of each step was divided into 50 bins, and the number of spikes occurring within each bin was accumulated. After division by the number of steps processed, this resulted in an averaged phase histogram for each animal which described the distribution of motor activity over a normalized step. The same was done for steps obtained when the treadwheel was held at the lower position and also for the unstimulated left middle leg. However, in the latter case, the onset of the 
depressor motoneurone's burst within each step was used as a trigger point because the movement of this leg was not recorded with a position detector.

For statistical analyses, the phase histograms of the individual animals were further processed with the aid of a statistics package (Statgraphics). The significance of differences in the phase histograms caused by stimulation was tested using $\chi^{2}$-tests. Application of circular statistics was not necessary because we divided the data into two groups and, for each group, compared the number of spikes obtained in two experimental situations.

\section{Results}

In the first experiment, the distance between the body and wheel was fixed at different heights and the forces with which the animal pulled or pushed the wheel in the vertical direction were measured. Because the animal could not influence the distance between its body and the wheel, this was an open-loop situation. The height was varied in $2 \mathrm{~mm}$ steps in the range $\pm 12 \mathrm{~mm}$ around the individual zero-force position for each animal. Each of these 13 position values was tested four times for each animal. The heights were chosen in random order. The results are shown in Fig. 1 (open circles). As mentioned in the Materials and methods section, the zero-force height of each animal was normalized to the value of $9 \mathrm{~mm}$.

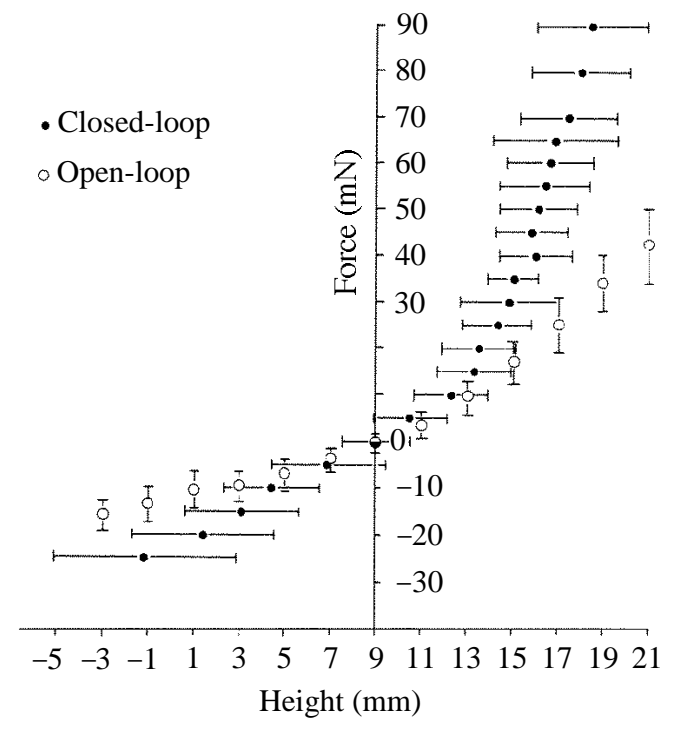

Fig. 1. The force-height characteristic of the open-loop system (open circles) and the closedloop system (filled circles). For the open-loop results, each point represents the result of 40 measurements (10 animals). In this experiment, the independent variable is height. For the closed-loop results, each point represents 30 measurements (10 animals, three walks each). In this experiment, the independent variable is load. The results are given as mean values \pm S.D. calculated for the 40 or 30 measurements, respectively. 
As the distance between body and wheel was increased, the animal tried to pull the wheel towards its body with more force. The increase in force is approximately linear for distances greater than $13 \mathrm{~mm}$, with a slope of $4.1 \mathrm{mNmm}^{-1}$. Correspondingly, as the distance became smaller than the zero-force distance, the legs produced forces that tended to push the wheel away from the body. Again the dependency was linear. The slope, however, was considerably less $\left(1.2 \mathrm{mNmm}^{-1}\right)$. The change in slope seemed to occur at a height between 11 and $13 \mathrm{~mm}$.

In this experiment (Fig. 1, open circles), the wheel was moved back to the zero-force position after each measurement. However, the results were no different when the test heights were approached from other positions. Other control experiments showed no significant hysteresis when the body height was not chosen randomly, but was serially increased and then decreased.

To estimate the dynamic properties of the height-control system, the temporal development of force was measured when the height of the wheel was changed in a steplike manner. This change was effected by moving the wheels down by 4 or $6 \mathrm{~mm}$ (thus increasing the body-wheel distance) or up by $6 \mathrm{~mm}$. The duration of the movement was approximately $0.3 \mathrm{~s}$ (range $0.15-0.45 \mathrm{~s}$ ). In addition, the reaction to a pulse-like change of height was investigated. In this case, the upward or downward movement of the wheel was immediately followed by the corresponding reverse movement. The duration of the pulse was approximately $0.6 \mathrm{~s}$ (range $0.45-0.8 \mathrm{~s}$ ). The force and the position of the wheel were recorded on the pen recorder. In total, 25 trials were obtained from six animals for each of the three step-like input functions, and 55 trials were obtained for the two pulselike functions. The results are shown in Fig. 2A-E. The input functions are shown only schematically. The force values were obtained in the following way: time $t_{0}$ refers to the time when the wheel position reached the end of the step, or the maximum of the pulselike function. Force values were taken every $50 \mathrm{~ms}$ for $2.5 \mathrm{~s}$ before and after $t_{0}$. The corresponding force values were used to calculate the mean and S.D. In Fig. 2, only some typical S.D. values are shown for clarity.

These results from walking animals do not exhibit the prominent phasic component of the step response shown in standing animals (e.g. Kemmerling and Varju, 1982; Cruse et al. 1989). We also found this phasic property when repeating our experiment, but with the animals simply standing on the wheel (not shown). There is a small overshoot about $0.7 \mathrm{~s}$ after the end of the step (Fig. 2A,D, arrows), but we cannot determine from our data whether this is significant. In any case, the prominent phasic response of the standing animals does not occur in the walking animal. Therefore, as a first approximation, the height-control system is a simple proportional system with few or no dynamic properties

Fig. 2. Dynamic properties of the height-control system under open-loop conditions. (A) Response to a step which increased the distance by $4 \mathrm{~mm}$. (B) Response to a $6 \mathrm{~mm}$ step in the same direction. (C) Response to a $6 \mathrm{~mm}$ step in the opposite direction. In all three cases, the data are mean values taken from 25 walks by six animals. (D,E) Responses to pulse-like input functions of $6 \mathrm{~mm}$ amplitude increasing or decreasing the distance, respectively. Mean values taken from 55 walks by six animals. The S.D. values are only shown for some selected cases. The input functions (height) are shown only schematically. The arrows mark the small overshoot at the end of a step. 

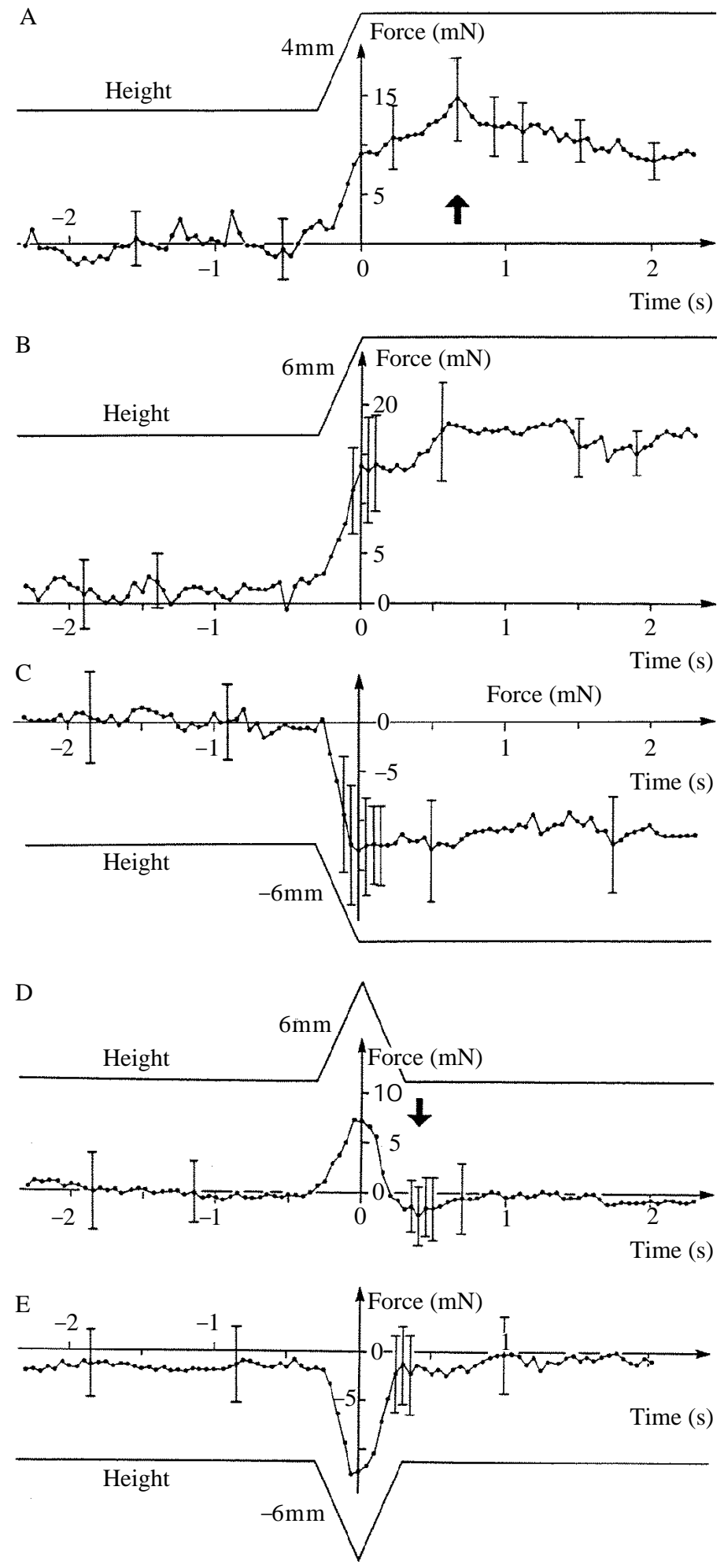

Fig. 2 
(i.e. it is a low-pass filter with a corner frequency greater than can be detected by our sampling rate of $50 \mathrm{~ms}$ ).

\section{Closed-loop experiments}

To test the responses of the height-control system under closed-loop conditions, the animal was permitted to adjust the distance between its body and the wheel. To investigate different loading situations, the wheel was loaded with different weights in the range $-25 \mathrm{mN}$ to $90 \mathrm{mN}$. The animals were found to walk sufficiently well with loads in this range. Negative weights tend to decrease the body-wheel distance. We measured the height adopted by the animal when it was loaded by each weight (Fig. 1, filled circles). In this case, the independent variable (load) is plotted along the vertical axis to enable a direct comparison with the data from the open-loop experiment. Ten animals were used, and each weight was applied three times for each animal. The data from the closed-loop experiment, like those from the open-loop experiment, can be approximated by two separate linear functions for values above and below a height of approximately $13 \mathrm{~mm}$. However, the slopes are different in both experimental situations $\left(2.2 \mathrm{mNmm}^{-1}\right.$ and $16 \mathrm{mNmm}^{-1}$ in the closed-loop experiments compared with $1.2 \mathrm{mNmm}^{-1}$ and $4.1 \mathrm{mNmm}^{-1}$ in the open-loop experiments).

In these experiments, the animals walked for about 10s without a weight before a new weight was applied. Control experiments showed that the results did not differ significantly when the weight applied between the measurements was $-10 \mathrm{mN}$ or $30 \mathrm{mN}$ instead of $0 \mathrm{mN}$.

\section{Contralateral coupling}

To test whether the reaction of a leg to different imposed heights in the open-loop experiment is also influenced by information from other legs, perhaps for the purpose of some compensatory reaction, the legs on both sides of the body were investigated separately. The axis of the left wheel was fixed at the zero-force height and then attached

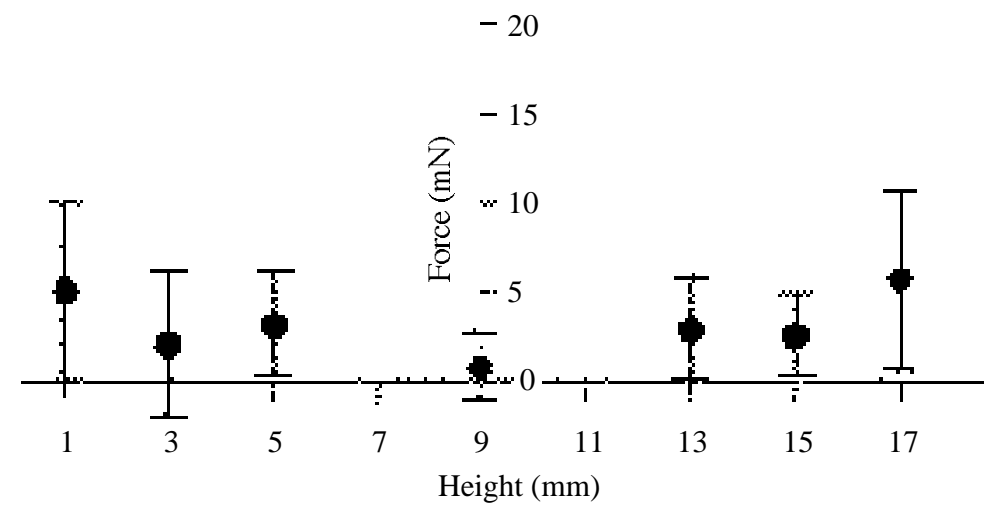

Fig. 3. Force-height characteristics of left legs when the height of the right wheel (abscissa) is changed under open-loop conditions. Each point represents the mean ( \pm S.D.) of 20 measurements (10 animals). 
to a force transducer. Only the right wheel could be moved up and down by the experimenter. The animals could still walk when the right wheel was moved in the range 1-17 mm. Greater changes prevented the animals from walking continuously on both wheels. As described for the open-loop experiment, the zero-force height was established for each animal individually. The height of the right wheel was then adjusted to different values in random order, and the forces produced by the left legs were measured (Fig. 3). The only clear effect was that the legs on the left side (walking on the fixed-height wheel) seemed to produce a small force which pulled the wheel towards the body, irrespective of whether the right legs were walking at a smaller or greater distance compared with the left side. Because the zero-force position was determined in the unstimulated situation (both wheels at the same height) before the actual experiment,

A

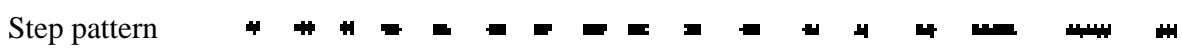

Wheel position

Right depressor of

Left depressor

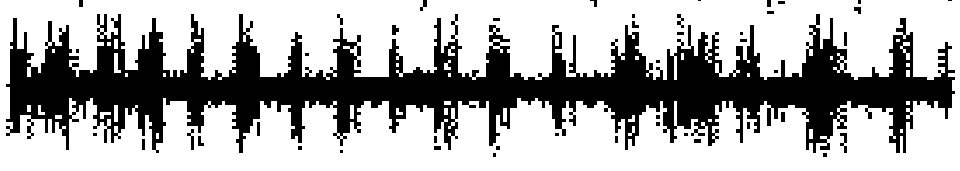

B

Leg position
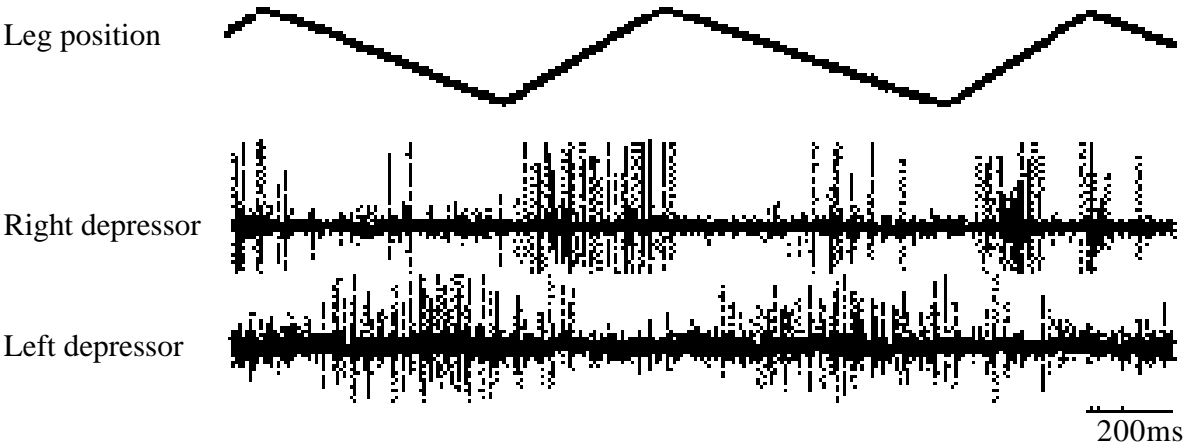

Fig. 4. Recordings of the right and left nerves (nl4a) containing the depressor trochanteris motoneurones of right (upper trace) and left (lower trace) middle leg during a normal walk on the treadwheel. (A) In the left-hand part, both legs walk on wheels set at the same body-wheel distance. The height of the right wheel is then lowered by $5 \mathrm{~mm}$ (wheel position). Swing movements of the right leg are indicated by bars (uppermost trace). (B) A section of the lefthand part of A, but using a larger time scale. The uppermost trace shows the position of the right leg. Upward deflections corresponds to swing movements. 
this result may reflect a general stimulation effect, as was found in standing animals (Cruse et al. 1989).

There were no interleg effects between walking legs and a 'standing leg of a walking insect'. In this experiment, one front leg stood on a separate force transducer while the other five legs walked on the wheel. The height of the wheel was then changed and the vertical forces produced by the front leg were measured. In this case there was again no dependency on the height of the wheel (i.e. of the other walking legs) (not shown). Because the front leg was not walking, this evidence is only indirect.

\section{Electrophysiological recordings}

In contrast to the results presented above, Bässler (1977) reported contralateral effects in a different experiment. We therefore repeated our experiment, but used electrophysiological recordings instead of force measurements because the former might provide a better resolution. The activity of the motoneurones innervating the thoracic parts of the depressor trochanteris muscle was investigated because the coxa-trochanter joint is mainly responsible for control of the body-ground distance. Only middle legs were considered.

\section{Activity pattern of depressor motoneurones during unstimulated walking}

The normal activity of motoneurones in nerve nl4a of the depressor trochanteris muscle of the middle leg in a walking animal is shown in Figs 4 and 5 (filled rectangles). The peak activity of the motoneurones occurred at about the middle of the swing, i.e. before the leg was moved downwards. The activity gradually decreased during the following stance phase and reached a minimum near the transition from stance to swing. (The decrease was found to be more rapid for right legs; compare Fig. 5A,B and see Discussion). During the stance, often only the motoneurones exhibiting smaller extracellular spike amplitudes were active, whereas during the swing, motoneurones with large spike amplitudes were also recruited. This pattern is in agreement with the leg movement, and with the findings of Epstein and Graham (1983) for the coxal part of the depressor trochanteris muscle. During the second half of the swing movement, the leg was lowered rapidly to reach the wheel. During the stance phase, a small, approximately constant, force was produced to maintain the body-wheel distance. The mean number of spikes per step was $50 \pm 29$ (across-animal average, mean \pm S.D., 219 steps). Step duration averaged $1.2 \pm 0.3 \mathrm{~s}$ ( $220 \mathrm{steps}$ ). During normal walking, the movements of the two middle

Fig. 5. Number of depressor trochanteris spikes per $23 \mathrm{~ms}$ bin during each step when the animal walked under different conditions. (A) Right middle leg. Filled rectangles, the height of the right wheel was the same as that of the left wheel (220 steps). Open rectangles, the height of the right wheel was lowered by $5 \mathrm{~mm}$ (169 steps). The beginning of the period corresponds to the anterior extreme position (AEP), i.e. the beginning of the stance phase. (B) Left middle leg. Filled rectangles, the height of both wheels was the same (241 steps). Open rectangles, the height of the right wheel was lowered by $5 \mathrm{~mm}$ (190 steps). The beginning of the period corresponds to the onset of the burst in the left leg depressor motoneurones. This roughly corresponds to the beginning of swing movements (see Fig. 4). 

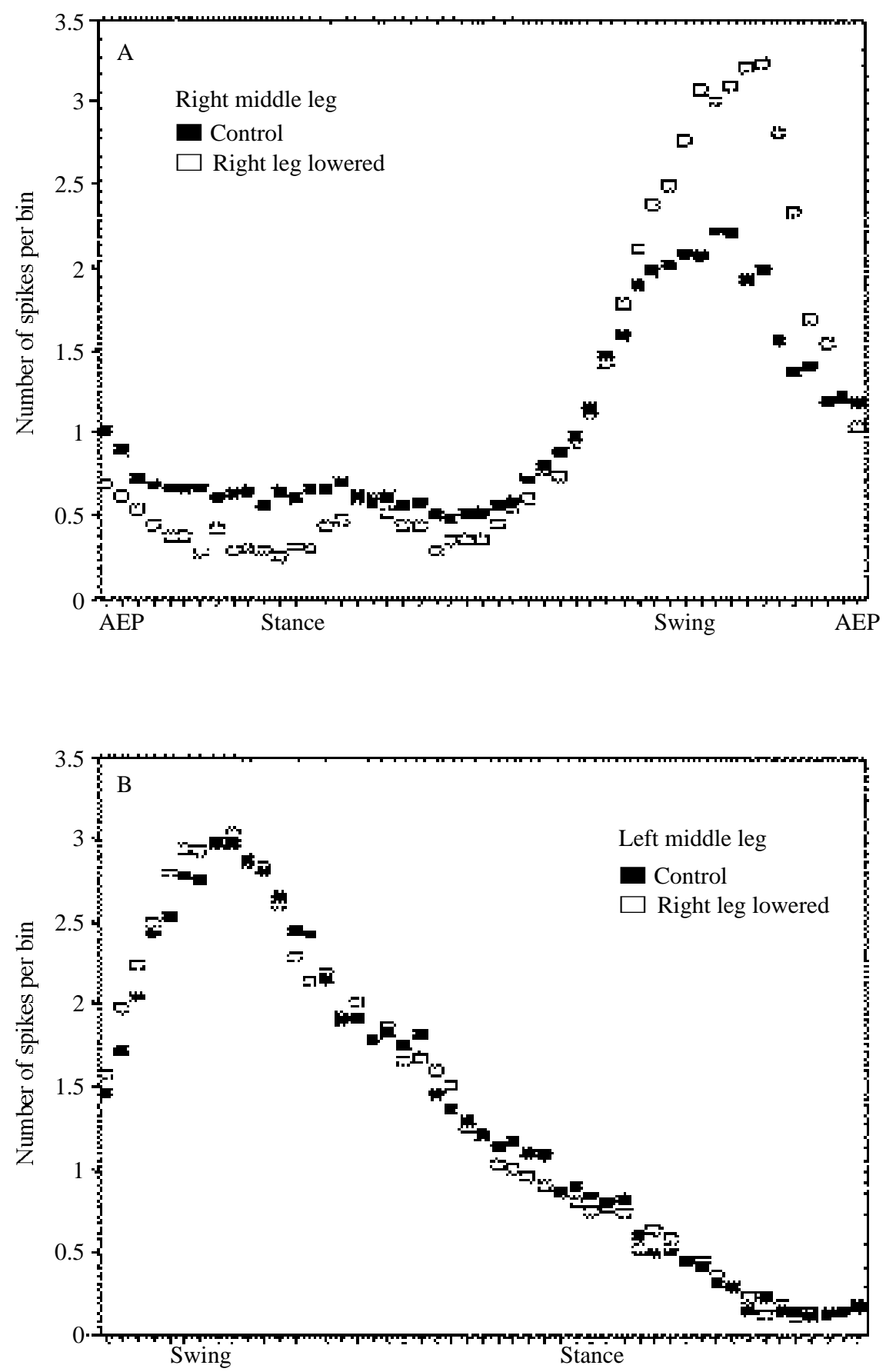

Bin number

Fig. 5 
legs were out of phase. This is reflected by the alternating activity shown in the nerve recording in Fig. 4.

\section{Changes in the motor activity pattern caused by variation in body height}

While the animal was walking, the right wheel was occasionally lowered by $5 \mathrm{~mm}$, but the animal continued to walk in a coordinated way, using the legs on both sides of the body even though they were at different heights. The step duration $(1.1 \pm 0.3 \mathrm{~s}, N=169$ steps) was not significantly affected by this stimulus $\left(\chi^{2}\right.$-test, $\left.P>0.1\right)$. The number of spikes per step also remained unchanged ( $51 \pm 31$ spikes, 192 steps, $\chi^{2}$-test, $\left.P>0.1\right)$.

There were, however, significant changes in the distribution of depressor trochanteris spikes within the step of the right middle leg. A comparison of the phase histograms for the two experimental situations shows that the activity of the right motoneurones significantly increased during the swing when the right legs were walking on the lowered treadwheel $\left(\chi^{2}\right.$-test, $\left.P<0.001\right)$ (open rectangles in Fig. 5A). In most cases, this was caused by increased activity of the motoneurones that had larger spike amplitudes. However, during stance, the activities of all right motoneurones were reduced when the right legs walked on the lower treadwheel $\left(\chi^{2}\right.$-test, $\left.P<0.001\right)$. During the swing movement, the activity of the motoneurones exhibiting the largest spikes (probably the fast and intermediate depressor motoneurones) was greater because the leg had to be moved farther down to reach the wheel. During stance on the lower wheel, all right depressor motoneurones generated fewer spikes. This qualitatively corresponds to the results of the force measurements. In contrast to this ipsilateral effect, no significant differences were found for legs contralateral to the lowered wheel (left legs). Fig. 5B shows the temporal distribution within a step of spikes in the left depressor trochanteris motoneurones (the height of the left wheel remained constant). As for the right side, there were no significant differences in the total number of spikes per step. These values were $72 \pm 35.5$ spikes $(N=205)$ when both wheels were set at the same height (step duration $1.1 \pm 0.3 \mathrm{~s}, N=241$ ) and $75 \pm 37$ spikes $(N=180)$ when the right wheel was lowered by $5 \mathrm{~mm}$ (step duration $1.2 \pm 0.3 \mathrm{~s}, N=190)$. There were no significant changes in the distribution of left depressor motoneurone spikes (Fig. 5B, $\chi^{2}$-test, $P>0.1$ ).

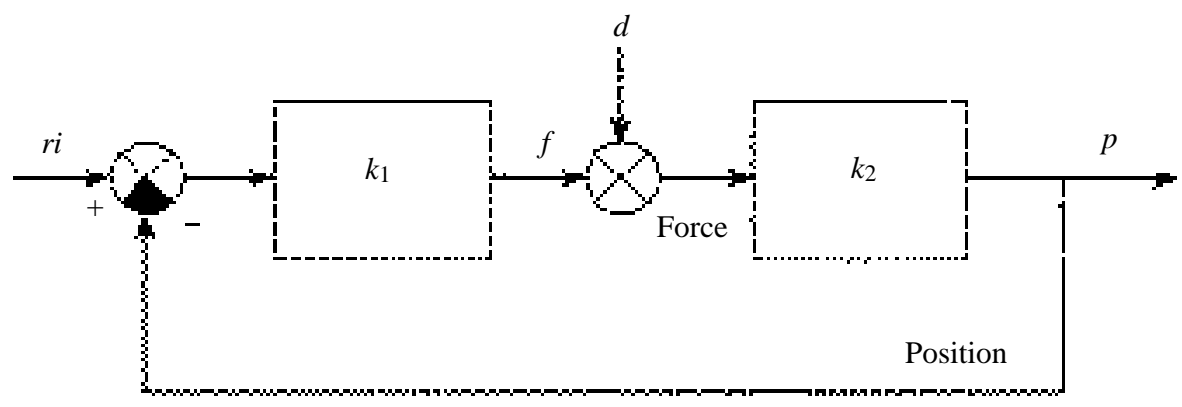

Fig. 6. A simple feedback controller. ri, reference input; $f$, actuator output (force); $d$, disturbance input (force); $p$, controlled variable (position); $k_{1}, k_{2}$, gain factors. For explanation see text. $k_{1}$ and $k_{2}$ have units of $\mathrm{mNmm}^{-1}$ and $\mathrm{mmmN}^{-1}$, respectively. 


\section{Discussion}

These experiments investigated the system which controls the body height of a walking animal. The open-loop and closed-loop results show that the system can be divided into two ranges, within each of which the system behaves approximately linearly. Because the weight of the animal corresponds to a force of about $8-10 \mathrm{mN}$, an animal walking upright on a horizontal plane has, according to the closed-loop results shown in Fig. 1 (filled circles), a body-ground distance of about $5 \mathrm{~mm}$. This distance would be about $12 \mathrm{~mm}$ if the animal walked hanging from a horizontal beam, when the gravity vector pointed in the opposite direction. The latter distance corresponds approximately to the height at which the gain change occurs. If we assume that the height-control system responds in the same way when the animal walks free on a horizontal plane or below a horizontal beam as it does when the animal walks in the closed-loop situation on the treadwheel, the following interpretation can be made. The average height during upright walking is within the linear range of that part of the force-height characteristic which has the smaller slope (gain factor), whereas during upside-down walking the average height is approximately at the position where the slope changes.

The difference in the slopes of the open-loop and the closed-loop results shows that, under closed-loop conditions, the height-control system exhibits less elasticity, i.e. greater rigidity, than it does under open-loop conditions. The static properties of a simple control system with negative feedback can be described by two gain constants, $k_{1}$ and $k_{2}$ (Fig. 6). The reference input is assumed to be constant and can therefore be set to zero for the following consideration. Under closed-loop conditions, the controlled variable $p$ (position) depends on the disturbing force $d$ according to $p=k_{\mathrm{c} l} d$, with the closed-loop gain $k_{\mathrm{cl}}=k_{2} /\left(1+k_{1} k_{2}\right)$ (see Cruse, 1981). Under open-loop conditions, the force output $f$ depends on position according to $f=-k_{1} p$. In our experiments, $k_{1}$ is about $1.2 \mathrm{mNmm}^{-1}$ for heights below $13 \mathrm{~mm}$. The corresponding closed-loop gain $k_{\mathrm{cl}}$ was $1 / 2.2=$ $0.46 \mathrm{mmmN}^{-1}$. Thus, $k_{2}$ can be calculated to be about $1.0 \mathrm{mmmN}^{-1}$. For heights above $13 \mathrm{~mm}$, the values are: $k_{1}=4.1 \mathrm{mNmm}^{-1}, k_{\mathrm{cl}}=0.06 \mathrm{mmmN}^{-1}$ and $k_{2}=0.9 \mathrm{mmmN}^{-1}$.

The dynamic properties of the height-control system are very simple. In contrast to the prominent phasic peak found in the standing animal, in the walking animal the system reacts tonically with a short time constant. This agrees with the results of Schmitz (1985), who investigated the corresponding reaction of the coxa-trochanter joint in the standing leg of the walking animal. The time constant is small enough to permit a reaction within one step.

We could not demonstrate any coupling between contralateral legs with respect to the height-control system. This agrees with findings in standing animals (Cruse et al. 1989). However, using a somewhat different walking situation, and with decerebrate animals, Bässler (1977) did find a contralateral compensatory reaction: in his experiments the distance between the insect's body and the ground was fixed by means of a special arrangement. When the legs on one side had to walk over a step, the legs on the other side showed some compensation, although there was no change of height on that side. Because of this disagreement, we performed the electrophysiological experiments in order to obtain a finer resolution than that provided by the force measurements. However, 
no significant influences on the contralateral legs were found in this case either. In a special experimental arrangement, the 'standing leg of a walking insect', the standing leg was not influenced by either contralateral or ipsilateral legs. Although these results cannot be unequivocally extrapolated to walking legs, they agree with those for standing animals, where there were no interleg influences on the height-control system between ipsilateral legs (Cruse et al. 1989). Two factors explain the difference between our results and those reported by Bässler. First, Bässler did not find significant effects for intact animals, so the compensatory response might only occur in decerebrate animals. Second, all three ipsilateral legs experienced the change in height simultaneously in our experiment, whereas in Bässler's experiment the three legs walked over the step sequentially.

The asymmetry in the form of the spike distributions within a step found between right and left legs was the same for all animals tested. Although we do not know the cause of this effect, we assume that, when the electrodes were placed, the animals were treated asymmetrically, which may have resulted in a slightly different behaviour of right and left legs. This, however, does not affect our conclusions.

We want to express our thanks to A. Baker for proofreading the English manuscript and to Professor Dr U. Bässler and Professor Dr J. Dean for helpful discussions. This work was supported by DFG grant (Cr 58/8-2).

\section{References}

BÄSSLER, U. (1977). Sensory control of leg movement in the stick insect Carausius morosus. Biol. Cybernetics 25, 61-72.

BÄssLeR, U. (1983). Neural Basis of Elementary Behavior in Stick Insects. Berlin, Heidelberg, New York: Springer.

CRUSE, H. (1976). The control of the body position in the stick insect (Carausius morosus), when walking over uneven surfaces. Biol. Cybernetics 24, 25-33.

CRuse, H.(1981). Biologische Kybernetik. Weinheim: Verlag Chemie.

Cruse, H., Dautenhahn, K. And Schreiner, H. (1992). Coactivation of leg reflexes in the stick insect. Biol. Cybernetics 67, 369-375.

CRuse, H. AND Müller, U. (1984). A new method of measuring leg position of walking crustaceans shows that motor output during return stroke depends upon load. J. exp. Biol. 110, 319-322.

CRuse, H., Riemenschneider, D. AND Stammer, W. (1989). Control of body position of a stick insect standing on uneven surfaces. Biol. Cybernetics 61, 71-77.

EPSTEIN, S. AND GRAHAM, D. (1983). Behaviour and motor output of stick insects walking on a slippery surface. I. Forward walking. J. exp. Biol. 105, 215-229.

Godden, D. H. AND GRAham, D. (1984). A preparation of the stick insect Carausius morosus for recording intracellularly from identified neurones during walking. Physiol. Ent. 9, 275-286.

GRAHAM, D. (1981). Walking kinetics of the stick insect using a low-inertia, counter-balanced, pair of independent treadwheels. Biol. Cybernetics 40, 49-58.

Graham, D. (1985). Pattern and control of walking in insects. Adv. Insect Physiol. 18, 31-140.

HerRmann, C. AND SCHMITZ, J. (1989). Proctolin enhances the contraction of the depressor trochanteris muscle in the stick insect. In Dynamics and Plasticity in Neuronal Systems. Proceedings of the 17th Göttingen Neurobiology Conference (ed. N. Elsner and W. Singer), pp. 129. Stuttgart, New York: Georg Thieme Verlag.

Kemmerling, S. AND VARJu, D. (1981). Regulation of the body-substrate distance in the stick insect: responses to sinusoidal stimulation. Biol. Cybernetics 39, 129-137. 
KEMMERLING, S. AND VARJU, D. (1982). Regulation of the body-substrate distance in the stick insect: step responses and modelling the control system. Biol. Cybernetics 44, 59-66.

MARQuardT, F. (1940). Beiträge zur Anatomie der Muskulatur und der peripheren Nerven von Carausius (Dixippus) morosus. Zool. Jb. Abt. Anat. Ont. Tiere 66, 63-128.

SCHMITZ, J. (1985). Control of the leg joints in stick insects: differences in the reflex properties between the standing and the walking states. In Insect Locomotion. Proceedings from the XVII International Congress of Entomology (ed. M. Gewecke and G. Wendler), pp. 27-32. Hamburg, Berlin: Paul Parey.

SchmiTZ, J. (1986). The depressor trochanteris motoneurones and their role in the coxo-trochanteral feedback loop in the stick insect Carausius morosus. Biol. Cybernetics 55, 25-34.

SchmitZ, J., Büschges, A. AND Delcomyn, F. (1988). An improved electrode design for en passant recording from small nerves. Comp. Biochem. Physiol. 91, 769-772.

Schmitz, J., BÜschgeS, A. AND KitTMAnN, R. (1991). Intracellular recordings from nonspiking interneurons in a semiintact, tethered walking insect. J. Neurobiol. 22, 907-921.

vON Helversen, O. AND Elsner, N. (1977). The stridulatory movements of acridid grasshoppers recorded with an opto-electronic device. J. comp. Physiol. 122, 53-64.

WENDLER, G. (1964). Laufen und Stehen der Stabheuschrecke: Sinnesborsten in den Beingelenken als Glieder von Regelkreisen. Z. vergl. Physiol. 48, 198-250.

Wood, D. W. (1957). The effect of ions upon neuromuscular transmission in a herbivorous insect. J. Physiol., Lond. 139, 119-139. 